\title{
Molecular-dynamics simulation of thermal conductivity in amorphous silicon
}

\author{
Young Hee Lee, ${ }^{*}$ R. Biswas, C. M. Soukoulis, C. Z. Wang, C. T. Chan, and K. M. Ho \\ Ames Laboratory, Microelectronics Research Center and Department of Physics, Iowa State University, Ames, Iowa 50011
}

(Received 11 June 1990; revised manuscript received 9 October 1990)

\begin{abstract}
The temperature-dependent thermal conductivity $\kappa(T)$ of amorphous silicon has been calculated from equilibrium molecular-dynamics simulations using the time correlations of the heat flux operator in which anharmonicity is explicitly incorporated. The Stillinger-Weber two- and three-body $\mathrm{Si}$ potential and the Wooten-Weaire-Winer $a$-Si model were utilized. The calculations correctly predict an increasing thermal conductivity at low temperatures (below $400 \mathrm{~K}$ ). The $\kappa(T)$, for $T>400$ $\mathrm{K}$, is affected by the thermally generated coordination-defect states. Comparisons to both experiment and previous calculations will be described.
\end{abstract}

\section{INTRODUCTION}

In spite of extensive studies, a number of outstanding problems do remain in understanding the lowtemperature properties of amorphous materials. Among the intriguing features have been the linear specific heat of glasses, which is generally believed to be due to the presence of localized two-level states. The nature of the vibrational modes in glasses and the excess vibrational density of states at low frequencies is also an interesting aspect. In this paper we study the thermal conductivity of amorphous materials, using amorphous silicon as a prototype.

The temperature-dependent behavior of the thermal conductivity is amorphous materials has the following three characteristic regimes. (1) At low temperatures $(T \leq 1 \mathrm{~K}), \kappa(T)$ is proportional to $T^{1.8-2.0}$, which was explained by Anderson, Halperin, and Varma ${ }^{1}$ as being due to the scattering from localized two-level states; (2) at intermediate temperature $(1 \leq T \leq 30 \mathrm{~K})$, a plateau was seen, which has attracted many theoretical investigations; ${ }^{2}(3)$ at high temperature $(T \geq 30 \mathrm{~K})$, where $\kappa(T)$ increases smoothly to a limiting value, in contrast to the crystalline insulators where it decreases with $1 / T$. Birch and Clark ${ }^{3}$ and $\mathrm{Kittel}^{4}$ gave qualitative explanations of regime (3) using the kinetic formula $\kappa=\mathrm{Cvl} / 3$ that is applicable only in the Boltzmann regime where one can assign velocities $v$ to propagating modes. Allen and Feld$\operatorname{man}^{5}$ (AF) have examined the validity of the kinetic formula in their recent calculation of the thermal conductivity in amorphous silicon. Cahill and $\mathrm{Pohl}^{6}$ argued, based on Einstein model, that regime (3) can be explained by locally uncorrelated harmonic oscillators with relaxation times of the order of the period of vibration. However, this model is only valid in the limit of highly disordered crystals. In this study we will study regime (3) with a different approach from previous works.

There exist many theoretical models for the structure of amorphous Si. A convenient theoretical model is the four-coordinated Wooten-Winer-Weaire ${ }^{7}$ (WWW) a-Si model. Biswas et al. ${ }^{8}$ obtained a vibrational density of states for the WWW model that agreed well with experiment. They have also obtained amorphous silicon configurations with molecular-dynamics (MD) simulations by cooling the molten silicon configuration. It is also known that Stillinger-Weber $(\mathrm{SW})$ potential $^{9}$ is valid for a wide range of properties of $a$-Si.

Since the WWW model has only four-coordinated silicon atoms, this will be a more convenient starting point for the thermal-conductivity calculations than molecular dynamics models of $a$-Si (Ref. 8 ) that have coordination defects. In addition, the use of the WWW model together with the SW Si potential allows a direct comparison with the recent calculation of Allen and Feldman.

Allen and Feldman ${ }^{5}$ have used the novel procedure of using the Kubo formula for calculating thermal conductivity in $a$-Si. Matrix elements of the heat-current operator were calculated between harmonic vibrational states. Although the zero-temperature WWW structure was used, the temperature entered through the quantum occupation of the vibrational states. AF obtained a $\kappa(T)$ that increases in temperature up to $300 \mathrm{~K}$ and then saturates at a value close to experiment. Low-temperature values $(T<300 \mathrm{~K})$ for $\kappa(T)$ were significantly smaller than experiment. We present in this paper an alternate approach that explicitly incorporates anharmonicities and accounts for temperature-dependent structural changes. Theoretical techniques developed by AF have served as a useful guide in the present work.

\section{THEORY}

Thermodynamic response functions are related to the appropriate time-correlation functions by the fluctuation-dissipation theorem. Specifically, the thermal conductivity is related to the time-correlation function of the heat-flux operator ${ }^{10} S(t)$,

$$
\kappa(T)=\int_{0}^{\infty} d t\langle\mathbf{S}(0) \cdot \mathbf{S}(t)\rangle .
$$

The heat-flux operator is defined by 


$$
S(t)=\frac{d}{d t} \sum_{i} \mathbf{r}_{i} \widetilde{E}_{i},
$$

where $\mathbf{r}_{i}$ is the position of the $i$ th atom and $\widetilde{E}_{i}=E_{i}(t)-\bar{E}_{i}$ is the excess site energy of the $i$ th atom. We have derived in the Appendix the expression for the heat flux in terms of the atomic variables to be

$$
S(t)=\sum_{i} \mathbf{v}_{i} \widetilde{E}_{i}+\sum_{i, l, i \neq l}\left(\mathbf{F}_{i l} \cdot \mathbf{v}_{i}\right) \mathbf{r}_{i l} .
$$

Here $\mathbf{v}_{i}$ is the velocity of the $i$ th site; $\mathbf{F}_{i l}$, the force on the $i$ th atom due to the $l$ th atom; $\mathbf{r}_{i l}$, the relative distance between $i$ and $l$ atoms. This holds for both two- and threebody interactions. We have assumed that the three-body energy $V_{i j k}$ can be assigned to the vertex atom $j$ for the site-energy calculations. The energy partition is not unique, however, since the temperature gradient varies on a scale much larger than interatomic distances, the details of the energy partition do not influence our results. The first term is the convection term which is a measure of atomic diffusion, and becomes appreciable only at higher temperatures. The second term describes the correlations between neighboring atoms and explicitly includes anharmonic effects. All quantities in Eq. (3) can be directly obtained from our molecular-dynamics simulations. This procedure of evaluating the thermal conductivity from equilibrium time-correlation functions is more well defined than the computation of a heat current produced by a temperature gradient in an amorphous network. Generally it is much more difficult to define appropriate boundary conditions or heat baths that would simulate a temperature gradient than using the equilibrium simulation performed here.

There are two ways of evaluating the integral of Eq. (1). One is a simple integration over some finite time. However, in practice the time correlations of $S(t)$ decay very slowly with time, leading to difficulty in estimating $\kappa(T)$ from the integral of Eq. (1). A way of avoiding this difficulty is to take the Fourier transform of the heat-flux operator defined by

$$
\mathbf{S}(\omega)=\int_{0}^{\infty} d t \mathbf{S}(t) e^{i \omega t}
$$

Then one can easily show that temperature-dependent thermal conductivity is the zero-frequency limit of the frequency dependent $\kappa(\omega, T)$,

$$
\kappa(T)=\left.\kappa(\omega, T)\right|_{\omega=0}=\frac{1}{3 V k_{B} T^{2}}|\mathbf{S}(\omega)|_{\omega=0}^{2}
$$

All variables in Eqs. (1)-(5) are purely classical quantities. It is important to take into account quantum corrections in the low-temperature regime in order to account for the different quantum occupation of phonon states from the classical Boltzmann distribution. We note that the energy of the classical oscillator in a MD simulation is different from that of a quantum oscillator in the real system. We correct this difference by requiring that the mean kinetic energy in the classical MD system is equal to that of the real quantum system, ${ }^{11}$

$$
k_{B} T_{\mathrm{MD}}=\int d \omega D(\omega) \hbar \omega\left(n+\frac{1}{2}\right),
$$

where $D(\omega)$ is phonon density of states and the phonon occupation number,

$$
n=\frac{1}{e^{\hbar \omega / k_{B} T}-1} .
$$

Thus the temperature is rescaled and the MD temperature is related to the quantum temperature. This relation is shown in Fig. 1(a) explicitly. At high temperature there is almost no difference between $T_{\mathrm{MD}}$ and $T$ so that the classical molecular dynamics directly provides the high-temperature limit. However, since the temperature regime we are mostly concerned with is below the Debye temperature (about $500 \mathrm{~K}$ for amorphous silicon), ${ }^{12}$ the temperature rescaling is necessary.

In addition to the temperature rescaling, the temperature gradient, $\partial T_{\mathrm{MD}} / \partial T$ multiplies the thermal conductivity since the gradient is implicitly associated with the thermal conductivity such that

$$
\dot{Q}=\kappa(\partial T / \partial x)=\kappa\left(\partial T_{\mathrm{MD}} / \partial x\right)\left(\partial T / \partial T_{\mathrm{MD}}\right)
$$

where $\dot{Q}$ is the heat flux. Thus the thermal conductivity is rescaled in addition to the temperature scaling. As shown in Fig. 1(b) the gradient decreases very rapidly at low temperature and this will be the dominant effect at this temperature regime. This is expected because the phonon occupation number increases as the temperature

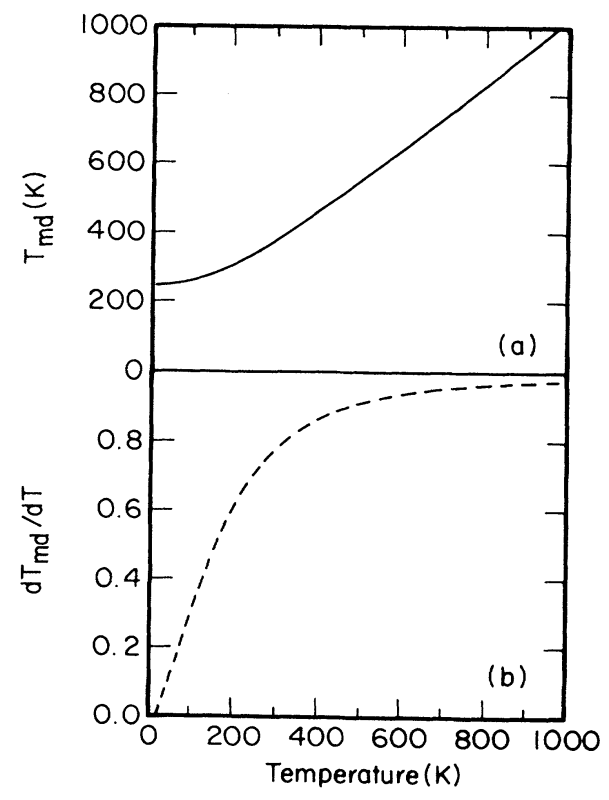

FIG. 1. (a) Relationship between classical temperature $T_{\mathrm{MD}}$ used in molecular-dynamics simulation and quantum temperature [Eq. (6)]. (b) Corresponding relation between temperature gradient of the classical temperature and quantum temperature. 
rises and thus this should increase thermal conductivity. The energy per particle is different in the classical and quantum cases, and so is the gradient of the energy or the derivative of the energy with temperature. As displayed in Fig. 1(b) this difference is largest in the lowtemperature regime, where the gradient correction plays a dominant role. This thermal effect is distinguished from the temperature-induced generation of defects, which will be discussed later. In fact, this gradient multiplied by $3 N k_{B}$ is equivalent to the heat capacity and this heat capacity increases slowly up to $1000 \mathrm{~K}$ even above the Debye temperature.

A complementary approach to the calculation of the thermal conductivity has been presented by AF, ${ }^{5}$ based on the Kubo-Greenwood formula. In that approach the eigenfrequencies and eigenvectors of the harmonic normal modes of the WWW $a$-Si network were obtained with the SW Si potential. The frequency-dependent thermal conductivity $\kappa(\omega)$ is related to the eigenfrequencies, occupation of eigenmodes, and matrix elements of the heatcurrent operator between the eigenstates. An advantage of this approach is the explicit inclusion of quantum statistics of the phonons, so that low-temperature properties are apparent. However, this approach neglects anharmonic interactions, which may be important at high temperatures. It is also based on using the zerotemperature relaxed static amorphous silicon network for calculations at all temperatures, which does not take into account changes in the structure or coordination defect densities with temperatures.

\section{RESULTS AND DISCUSSION}

\section{A. Crystalline fcc lattice}

In order to test our method, we calculated the thermal conductivity of a fcc crystal and compared to the results obtained previously by Ladd, Moran, and Hoover (LMH). ${ }^{13}$ As in the work of LMH we used a purely repulsive inverse-twelfth-power pair potential. The time correlation of $S(t)$ [Eqs. (1-3)] decayed sufficiently rapidly that the integral of Eq. (1) of the correlation function was well behaved and could be evaluated directly to obtain the thermal conductivity at two temperatures of the order of $\frac{1}{2} T_{m}$, where $T_{m}$ is the melting temperature (Fig. 2 ). Since the temperature was high enough, the temperature and thermal conductivity rescaling were unnecessary. We have also verified that the time integral of Eq. (1) was consistent with extrapolation of $\kappa(\omega, T)$ [Eq. (5)] to zero frequency, verifying the validity of our computational procedure.

As expected for crystalline materials, the hightemperature thermal conductivity decreases with temperature, due to the increase of the anharmonic effects with increasing temperature. We now consider the case of amorphous silicon for which the behavior is quite different.

\section{B. Amorphous silicon}

We have considered the four-coordinated WWW amorphous-Si configuration that has 216 atoms in a cubic

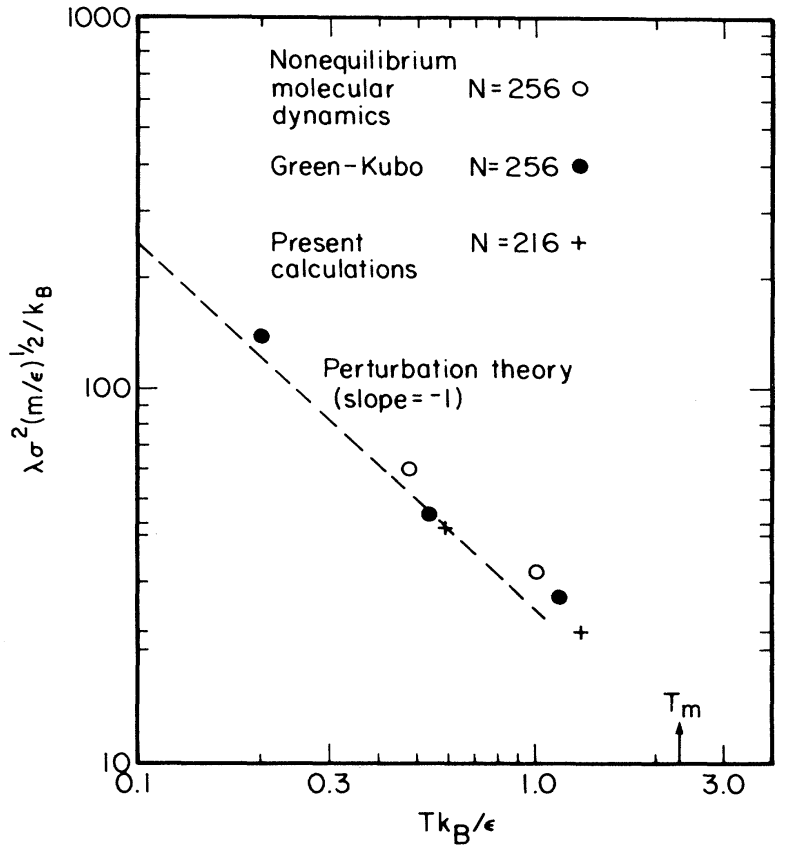

FIG. 2. Thermal conductivity vs temperature of fcc crystal with the simple repulsive potential. + are our calculations. Points as well as the dotted line are from Ref. 14. $T_{m}$ is the melting temperature. Dimensionless units have been used.

supercell of $16.13 \AA$. The WWW model was relaxed by a steepest-descent calculation to a local minimum of the SW potential. The WWW model was then equilibrated with the SW potential at various temperatures with runs of 20000-40000 time steps, using a time step $\tau=0.74 \times 10^{-15} \mathrm{~s}$ where energy conservation was achieved up to eight significant figures. For each temperature $T$, molecular dynamics runs of 65536 steps were employed to calculate the time correlations of $S(t)$ and $\kappa(\omega)$. The decay of the time-correlation function $\langle S(t) S(0)\rangle$ was very slow, making the direct evaluation of Eq. (1) unfeasible. Hence we evaluated $\kappa(\omega)$ from Eq. (5) and extrapolated the calculation to the zero-frequency limit (Fig. 3) for various temperatures.

The frequency-dependent thermal conductivity for amorphous silicon for various temperatures (Fig. 3) incorporates the quantum corrections described in Eqs. (6) and (7). These corrections renormalize both the effective temperature and the magnitude of the thermal conductivity. Without quantum corrections, a much higher value of the low-temperature thermal conductivity would be obtained. Each curve is the average over several runs of 65536 time-step runs. $\kappa(\omega)$ decays rapidly at high frequencies, with the decay much faster than the result of AF. This is probably due to the inclusion of the anharmonic effects in our calculation, as opposed to the harmonic approximation previously employed. It is also plausible that $\kappa(\omega)$ should decrease with increasing frequency, since high-frequency thermal perturbations should not lead to thermal current transport. We have 


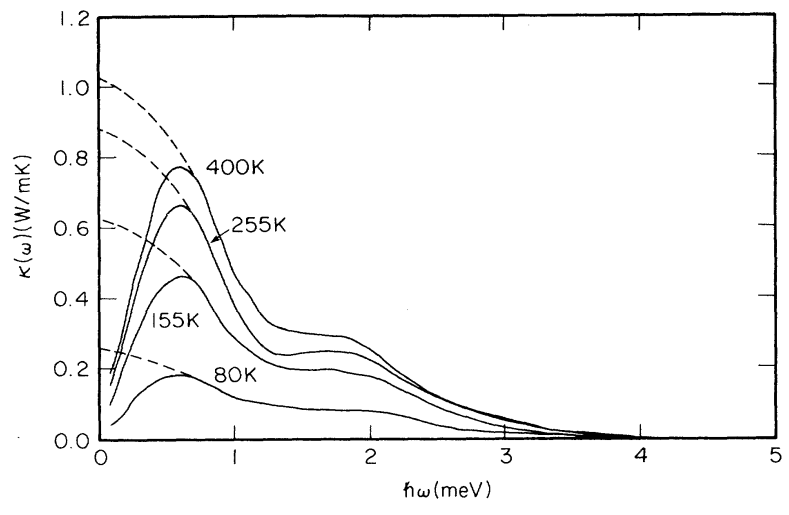

FIG. 3. Calculated frequency-dependent thermal conductivity for temperatures $80,155,255$, and $400 \mathrm{~K}$. Dotted lines show the extrapolation needed to obtain the static thermal conductivity at different temperatures.

studied the decay of $\kappa(\omega)$ by plotting $\kappa(\omega)$ as a function of $\omega$ and find that $\kappa(\omega) \sim \omega^{-4.1}$ to $\omega^{-3.8}$, for the highfrequency regime of $2-4 \mathrm{meV}$ in Fig. 3. This suggests that Rayleigh scattering with a decay of $\omega^{-4}$ is a valid representation of the high-frequency regime. This Rayleigh scattering has been suggested by other authors. ${ }^{15}$

The absence of long-wavelength modes due to the finite size of the simulation box in our calculation severely depletes the contribution to the static thermal conductivity and leads to the dip in the calculated $\kappa(\omega)$ at low frequency. The $\kappa(\omega)$ whose mean free path is less than the system size $(16.13 \AA)$ is explicitly included in our timecorrelation function of heat-flux operator [Eq. (3)]. However, the thermal propagating modes that are not scattered by the phonons and have a mean free path greater than the system size, have been lost due to the finite system size. In principle if we used an infinite size simulation system, the low-frequency dip would vanish. The position of our peak in $\kappa(\omega)$ is similar to the result of AF and is approximately independent of temperature.

The dip of $\kappa(\omega)$ at low frequencies below $1 \mathrm{meV}$ is also associated with two other practical limitations in MD simulation. Firstly, the finite time of the computer run (48.5 ps) leads to a cutoff frequency of $0.09 \mathrm{meV}$. Second$1 y$, the finite size of the system leads to a cutoff frequency of approximately $6 \mathrm{meV}$, below which there are no extended modes. If the size of the system is bigger, we would have higher peaks. This was confirmed by AF. ${ }^{16}$ In the limit of an infinite size system and an infinite simulation time, peak of $\kappa(\omega)$ would occur at exactly $\omega=0$. AF have argued that the $\kappa(\omega)$ curve may be smoothly extrapolated to zero frequency to obtain a reasonable theoretical estimate for the static thermal conductivity. We adopt this ansatz to obtain the temperature dependent $\kappa(T)$ in Fig. 4. We also estimate numerical errors both from the extrapolation procedure and from the finite time runs, and display these in Fig. 4.

Illustrated in Fig. 4 is the temperature-dependent behavior of the thermal conductivity which was obtained from the extrapolation of frequency-dependent thermal

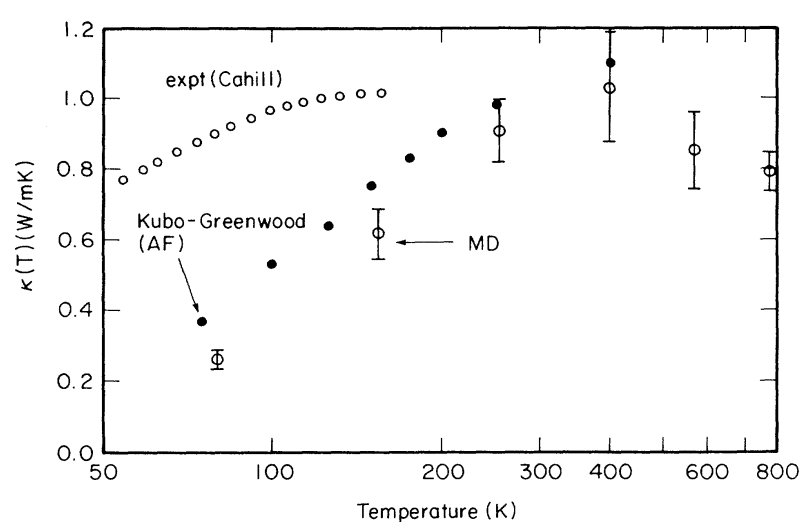

FIG. 4. Thermal conductivity vs temperature for amorphous silicon. Solid circles are from Allen and Feldman (Ref. 5) and open circles are from experiment (Ref. 17). Open circles with error bars are the present calculations.

conductivity in Fig. 3 together with values derived by $\mathrm{AF}$, and experimental values by Cahill et al. ${ }^{17}$ The experimental values of $\kappa(T)$ show a gradual increase from 50 to $150 \mathrm{~K}$ and indicate a saturation of $\kappa(T)$ for higher temperature. The physics of the experimentally observed saturation at high $T$ for glasses is that the phonons are so strongly scattered at high $T$ that their mean free path reaches a limiting lower value of the order of an interatomic spacing $a$, leading to a saturation value of the thermal conductivity at $\kappa \sim(1 / 3) C_{v} v a$. This is very unlike the case for crystals where the mean free path $l$ is much larger and decays as $1 / T$ at high $T$, due to increasing phonon-phonon scattering, leading to $\kappa(T) \propto 1 / T$ at high $T$.

Our calculated value of $\kappa(T)$ rises slowly with temperature, reaches a maximum near $300 \mathrm{~K}$ and shows a small decrease above $400 \mathrm{~K}$. The essential behavior of the feature is basically similar to the experiment ${ }^{17}$ and also to previous theoretical work. ${ }^{5}$ In this temperature regime, the static phonon mean free paths $l$ can be assigned to its maximum value which is the phonon wavelength $\lambda$ or the scale of disorder, ${ }^{18}$ while the heat capacity still increases and reaches a saturation value at high temperature. Thus the thermal conductivity basically follows the heat capacity which was explicitly introduced in Fig. 1(b). This effect must be emphasized since it was not clear from both the experiment and the theoretical calculation. In addition, our results are consistently lower than AF's. This is expected since our MD simulation includes anharmonicity. The anharmonicity leads to more phononphonon scattering and lowers the thermal conductivity, relative to values of AF. This anharmonicity is expected to be larger at higher temperature.

An aspect that needs discussion is the decrease of the calculated $\kappa(T)$ above $400 \mathrm{~K}$. The thermal effect of increasing occupation of phonon modes is expected to lead to an increase in the thermal conductivity for higher temperatures. This implies that there must be another mechanism affecting the thermal conductivity. In our MD simulation, the WWW $a$-Si configuration was equilibrat- 
ed by the SW Si potential at various temperatures. We explicitly found temperature-induced structural changes. To illustrate the structural changes, we performed a steepest-descent quench to the nearest local minimum, for equilibrated configurations at the various temperatures. This led to an inherent structure ${ }^{9}$ that eliminates the effects of thermal disorder. The potential energy of these inherent configurations, Fig. 5(a), shows a decrease with temperature, indicating the thermal annealing of the WWW model. Also the number of coordination defects increases with temperature. There is an increase in both the number of dangling bonds (three-coordinated atoms) [Fig. 5(b)] and the number of floating bonds (fivecoordinated atoms) [Fig. 5(c)] with temperature. In fact the WWW model has only one dangling bond and one floating bond at low temperatures (below $100 \mathrm{~K}$ ). The number of floating bonds is approximately twice that of dangling bonds. The over-coordinated $a$-Si model from the higher temperature runs is very similar to the slow melt-quenched model generated by Luedtke and Landman. ${ }^{19}$ We also observed that the location of the coordination defects changes with temperature, suggesting defect diffusion. The temperature-induced coordination defects act as new scattering centers for phonons and lead to the decrease in thermal conductivity above $400 \mathrm{~K}$. We refer to this as the defect effect. ${ }^{20}$

In addition to the increase in defect densities above 400 $\mathrm{K}$, we need to consider the alternative mechanism of increasing phonon-phonon scattering at higher $T$ which can also lead to decreasing $\kappa(T)$. We take into account here two sources for the phonon lifetimes. These are (i) scattering from static structural disorder with a lifetime $\tau_{1}$ - the analogue of impurity scattering in crystals, and (ii) phonon-phonon scattering with a lifetime of $\tau_{2}$, which is temperature dependent. If the average phonon lifetime from both processes is $\tau$, then

$$
\tau^{-1}=\tau_{1}^{-1}+\tau_{2}^{-1}
$$

The scattering or thermal transport is dominated by the scattering process with a shorter lifetime.

In crystalline materials one expects the impurity contribution $\tau_{1}$ to the lifetime to be much larger than $\tau_{2}$ at room temperature, so that the high-temperature $\kappa$ is governed by phonon-phonon interactions. Alternatively, in strongly disordered systems, such as $a$-Si, one expects $\tau_{1}<\tau_{2}$ at room temperature, i.e., much stronger scattering from the static structural disorder - an inference also suggested by the calculation of AF. The harmonic AF calculation includes $\tau_{1}$ processes but neglects anharmonicity or $\tau_{2}$ scattering processes and yet reproduces reasonable values of $\kappa(T)$ for $T \sim 300-400 \mathrm{~K}$. This argues for $\tau_{1}<\tau_{2}$ and implies that disorder scattering lifetimes can account for the thermal conductivity of $a$-Si at 300-400 $\mathrm{K}$. Also the mean free path $v \tau$ has not reached its lower limit (of the interatomic spacing) yet at $400 \mathrm{~K}$ since $\kappa(T)$ is still increasing. Since $\tau_{1}<\tau_{2}$ an increase in density of coordination defects (as in Fig. 5) should decrease $\tau_{1}$ and decrease $\kappa(T)$ - with the defect centers having a larger effect in decreasing $\kappa$ than the phonon-phonon scattering.

We have thus far found that there exist two competing processes in the system, the thermal effect and the coordination defect states. At low temperature, the thermal conductivity increases due to the fact that the thermal effect increases the heat capacity. The quantum corrections are necessary. Thus the thermal effect is dominant at the low-temperature regime. At high temperature above $400 \mathrm{~K}$, the coordination defects increase as shown in Fig. 5 and reduce the thermal conductivity. However,

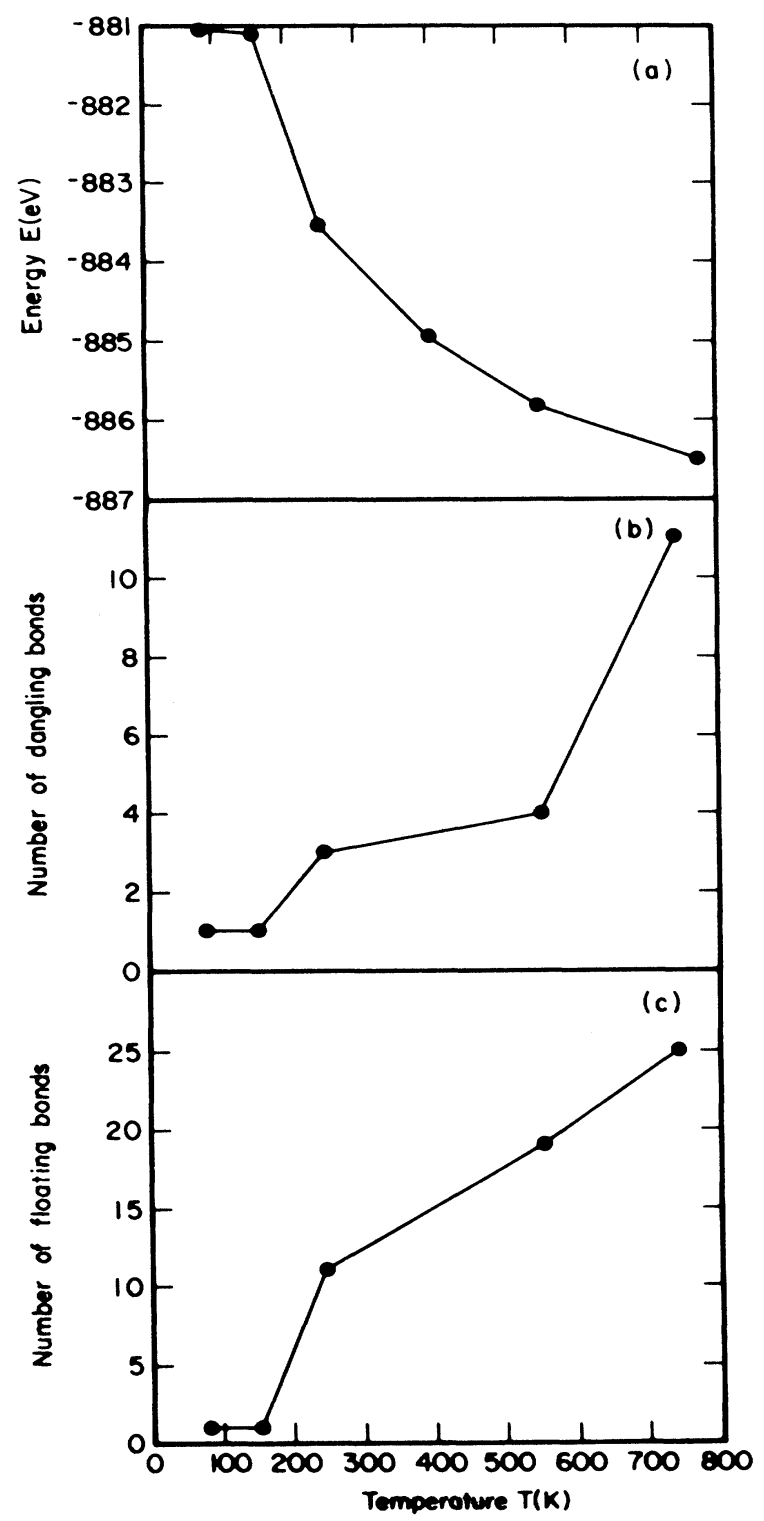

FIG. 5. All configurations were obtained by a steepestdescent quench of $a$-Si models, equilibrated at various quantum temperatures. (a) shows the potential energies of the configurations, whereas (b) and (c) show the increasing number of dangling-bond and floating-bond defects. 
in this case the increase in defect states dominates the thermal effect and thus the thermal conductivity decreases. The coordination defects are a property of the SW Si potential. A similar high density of floating bonds has also been observed in melt-quenched $a$-Si models generated by Luedtke and Landman. ${ }^{19}$ The decrease in $\kappa(T)$ above $400 \mathrm{~K}$ is mainly a consequence of these coordination defects, and was not present in the AF calculation. Beyond $1100 \mathrm{~K}$ the amorphous network may melt and the thermal conductivity decreases further.

Our calculated values of the thermal conductivity are lower than those of experiment in the low-temperature regime 50-200 K, but they approach values in good agreement with experiment at $300-400 \mathrm{~K}$. In fact, the classical MD simulations are supposed to yield the correct high-temperature limit. The discrepancy between experiment and theory at low temperatures is due to the absence of long-wavelength propagating modes in our model that have mean free paths longer than our simulation cell size, and hence can efficiently transport heat. However, we do account for long-wavelength modes (below $6 \mathrm{meV}$ ) that have mean free path smaller than the size of the cell. We expect a better comparison with experiment can be achieved in simulations using a larger cell.

We can make a simple estimate of the thermal conductivity arising from the missing long-wavelength modes. Generally the thermal conductivity $\kappa(T)$ can be written as

$$
\kappa(T)=\frac{1}{3}\left[\int_{0}^{\omega_{c}}+\int_{\omega_{c}}^{\infty}\right] d \omega D(\omega) C_{v}(\omega) l(\omega) v(\omega)
$$

The cutoff frequency $\omega_{c}$ is $6 \mathrm{meV}$ in our simulation model. Here $D(\omega)$ is the density of vibrational states, $C_{v}$ is the specific heat, $l(\omega)$ is the mean free path and $v(\omega)$ the velocity of the modes with frequency $\omega$. The contribution above $\omega_{c}$ has already been considered in the simulation. One way to estimate this contribution was proposed by AF by approximating $l(\omega)$ to be either a constant or $\sim \omega^{-1}$, and this approach fits the high-temperature limit but the calculated $\kappa(T)$ is considerably lower than experiment below $150 \mathrm{~K}$.

We may estimate the contribution of (9) below $\omega_{c}$ by assuming a $D(\omega) \sim \omega^{2}$ and assuming that $l(\omega) \sim l_{0}\left(\omega_{\min } / \omega\right)^{4}\left(=l_{\text {Ray }}\right)$, i.e., Rayleigh scattering for frequencies $\omega_{\min }<\omega<\omega_{c}$. More generally we should include the contributions from two-level states and boundary scattering which have mean free paths $l_{\mathrm{TLS}}$ and $l_{B}$, respectively. Then

$$
l^{-1}(\omega)=l_{\mathrm{TLS}}^{-1}+l_{\mathrm{Ray}}^{-1}+l_{B}^{-1}
$$

The contribution $l_{\text {TLS }}$ from two-level states varies as $\omega^{-1}$, whereas $l_{B}$ is a constant, independent of frequency. For our estimate of $\kappa(T)$ we neglect contributions from the two-level states and the boundary scattering for the following reasons: (i) The two-level states lead to extra scattering at low frequencies that may be approximately accounted for by redefining the $\omega_{\min }$ parameter. (ii) To the best of our knowledge, a plateau in the thermal conductivity has not been observed in $a$-Si and hence the density of two-level states is not expected to be significant. (iii) Including either two-level states or boundary scattering introduces further unknown parameters.

Hence for the estimate of the low-frequency modes to the thermal conductivity we include just the Rayleighscattering contribution that involves the scattering by local-density fluctuations or bond-length fluctuations of the system, which alter the local sound velocities. The Rayleigh scattering has also been inferred by other authors. ${ }^{2,15}$ We find that we can integrate (9) from $\omega_{\min }$ to $\omega_{c}$ to account for the experimental value of $\kappa(T)$ at $40 \mathrm{~K}$ $[\kappa(40 \mathrm{~K})=0.62 \mathrm{~W} / \mathrm{mK}]$, if we assume values of $\omega_{\min }=0.4$ $\mathrm{meV}$, and $l_{0}=4.1 \times 10^{-5} \mathrm{~m}$, and use an average sound velocity of $8.4 \times 10^{3} \mathrm{~m} / \mathrm{s}$ from crystal Si data. Similar values of $\omega_{\min }$ and $l_{0}$ have been inferred for several glasses by Graebner, Golding, and Allen (GGA), ${ }^{2}$ from fits of the low temperature $\kappa(T)$ to experimental data. This suggests that our inferred values are physically reasonable and consistent with previous estimates. ${ }^{2}$ This simple analysis indicates that the long-wavelength modes with large mean free paths significantly contribute to the low-temperature thermal conductivity in $a-\mathrm{Si}$. A full theory of the low-temperature $\kappa$ must incorporate (i) physically reasonable temperature dependences of $\omega_{\min }$ and $l_{v}$, and (ii) merge satisfactorily with the hightemperature $\kappa$ already calculated. These are aspects for further work.

Our calculations may be extended in a straightforward way to amorphous germanium $(a-\mathrm{Ge})$, which is considered to have an amorphous network and structural disorder very similar to $a-\mathrm{Si}$. The main difference is the heavier mass, which leads to a softening of the entire vibrational density of states by $1 / \sqrt{M}$ which is 0.62 from the $a$-Si values. The lower-frequency phonons directly renormalize the thermal conductivity by the same amount so that $\kappa(T)$ experimentally saturates at $\simeq 0.6$ $\mathrm{W} \mathrm{m}{ }^{-1} \mathrm{~K}^{-1}$ for $a-\mathrm{Ge} .{ }^{17}$

\section{CONCLUSIONS}

In summary, we have calculated the thermal conductivity of amorphous silicon as a function of temperature using equilibrium molecular-dynamics simulations. Our method is based on relating the thermal conductivity to the time-correlation function of the heat-flux operator. We have derived a form for the heat-flux operator in terms of interatomic positions, velocities, and forces, that is appropriate for both two- and three-body interatomic potentials. The expressions for the thermal conductivity Eqs. (1)-(5) may be applied directly to other physical properties. To the best of our knowledge, this is the first demonstration that thermal conductivity may be calculated from molecular-dynamics simulations for systems described by many-body potentials.

We have presented detailed results for the temperature-dependent thermal conductivity of amorphous silicon and limited results for fcc metals. Our calculations for $a$-Si are in the temperature range 50 to 800 
$\mathbf{K}$, above temperatures where either two-level states or a plateau in thermal conductivity may be important. We demonstrate that in this temperature range, the thermal conductivity $\kappa(T)$ of $a-\mathrm{Si}$ increases with temperature, whereas the thermal conductivity of an fcc crystal decreases with temperature consistent with experimental results. The increase in $\kappa(T)$ is typical for most amorphous solids and due to an increase in the vibrational modes that can transport heat current. The decrease in $\kappa(T)$ for crystalline materials is related to the decreasing phonon mean free paths with temperature.

Our results for $a$-Si in the $50-400 \mathrm{~K}$ range agree well with previous calculations of AF using the KuboGreenwood formula. Our calculated values are lower than those of AF, consistent with the inclusion of anharmonicity in the present calculations. The present calculations indicate a decrease in $\kappa$ above $400 \mathrm{~K}$ due to the increasing number of coordination defects generated at higher temperature.

The calculated values of $\kappa(T)$ are still lower than experiment between $50-200 \mathrm{~K}$, but approach experimental values in the $300-400-K$ range. We emphasize that the absence of long-wavelength propagating phonon modes in our calculations, with mean free paths larger than our cell size, accounts for the difference between calculated values and experiments at lower temperatures. Larger simulation systems may be needed for better comparison with experiment.

\section{ACKNOWLEDGMENTS}

We wish to thank J. Feldman, P. B. Allen, and R. O. Pohl for helpful discussions and suggestions. We acknowledge support from the National Science Foundation under Grant No. DMR-8819379. We acknowledge a National Science Foundation grant of supercomputer time at the National Center for Supercomputing Applications, Champaign, Illinois. Ames Laboratory is operated by Iowa State University for the U.S. Department of Energy (U.S. DOE) under Contract NO. W-7405-ENG-82 and is supported by the director for Energy Research,
Division of Material Sciences, U.S. DOE. One of us (Y.H.L.) would like to thank Ames Laboratory and Microelectronics Research Center for their hospitality and support during this project, and acknowledge support from the Korea Science and Engineer Foundation and Ministry of Education of Korea.

\section{APPENDIX}

In this appendix we derive the expression for the heatflux operator [Eq. (3)]. Starting from the definition of the heat-flux operator $S(t)$ [Eq. (2)], we express $S(t)$ as

$S(t)=\sum_{i} \mathbf{v}_{i} \widetilde{E}_{i}+\sum_{i} \mathbf{r}_{i}\left(\sum_{l} \mathbf{v}_{l} \cdot \frac{\partial E_{i}}{\partial \mathbf{r}_{l}}+\sum_{l} \mathbf{F}_{l} \cdot \frac{\partial E_{i}}{\partial \mathbf{p}_{l}}\right)$.

Here the time derivative has been reexpressed as a derivative of the positions and velocities. We introduce here the force on atom $l$ due to atom $i, \mathbf{F}_{l i}=\partial E_{i} / \partial \mathbf{r}_{l}$, and write $S(t)$ as

$S(t)=\sum_{i} \mathbf{v}_{i} \widetilde{E}_{i}+\sum_{i, l}-\left(\mathbf{F}_{l i} \cdot \mathbf{v}_{l}\right) \mathbf{r}_{i}+\sum_{i}\left(\mathbf{F}_{i} \cdot \mathbf{v}_{i}\right) \mathbf{r}_{i}$,

which is equivalent to

$$
S(t)=\sum_{i} \mathbf{v}_{i} \widetilde{E}_{i}+\sum_{i, l}\left(-\mathbf{F}_{l i} \cdot \mathbf{v}_{l}+\mathbf{F}_{i l} \cdot \mathbf{v}_{i}\right) \mathbf{r}_{i} .
$$

The $i=1$ term vanishes in the summation. We may then interchange the $i$ and $l$ indices of the second term to get

$$
S(t)=\sum_{i} \mathbf{v}_{i} \widetilde{E}_{i}+\sum_{i, l, i \neq l}\left(\mathbf{F}_{l i} \cdot \mathbf{v}_{l}\right)\left(\mathbf{r}_{l}-\mathbf{r}_{i}\right) .
$$

The first term is the convection term describing atomic diffusion and is expected to be significant only at high temperatures near melting. The second term describes the correlations between neighboring atoms, including anharmonic effects. Equation (14) is easier to interpret physically. The thermal current from the second term directly depends on the scalar product of the velocity $\mathbf{v}_{l}$ of atom $l$ and the force $\mathbf{F}_{l i}$ exerted by atom $i$ on atom $l$, consistent with the physical picture of heat transport. Equation (14) also provides the correct direction of heat transport and is equivalent to Eq. (3) in the text.
${ }^{*}$ Permanent address: Department of Physics, Jeonbug National University, Jeonju, Jeonbug 560-756, Republic of Korea.

${ }^{1}$ P. W. Anderson, B. I. Halperin, and C. M. Varma, Philos. Mag. 25, 1 (1972); W. A. Phillips, J. Low. Temp. Phys. 7, 351 (1972).

${ }^{2}$ For instance, C. C. Yu and J. J. Freeman, Phys. Rev. B 36, 7620 (1987); J. E. Graebner, B. Golding, and L. C. Allen, ibid. 34, 5696 (1986).

${ }^{3}$ F. Birch and H. Clark, Am. J. Sci. 238, 529 (1940).

${ }^{4}$ C. Kittel, Phys. Rev. 75, 972 (1948).

${ }^{5}$ P. B. Allen and J. L. Feldman, Phys. Rev. Lett. 62, 645 (1989).

${ }^{6}$ D. G. Cahill and R. O. Pohl, Solid State Commun. 70, 927 (1989).

${ }^{7}$ F. Wooten, K. Winer, and D. Weaire, Phys. Rev. Lett. 54, 1392 (1985); K. Winer, Phys. Rev. B 35, 2366 (1987).

${ }^{8}$ R. Biswas, A. M. Bouchard, W. A. Kamitakahara, G. S. Grest, and C. M. Soukoulis, Phys. Rev. Lett. 60, 2280 (1988).

${ }^{9}$ F. H. Stillinger and T. A. Weber, Phys. Rev. B 31, 5262 (1985).

${ }^{10}$ D. A. Mcquarrie, Statistical Mechanics (Harper and Row, New York, 1976).

${ }^{11}$ C. Z. Wang, C. T. Chan, and K. M. Ho, Phys. Rev. B 42, 11276 (1990).

${ }^{12}$ M. Mertig, G. Pompe, and E. Hegenbarth, Solid State Commun. 49, 369 (1984).

${ }^{13}$ A. J. C. Ladd, B. Moran, and W. G. Hoover (unpublished).

${ }^{14}$ J. J. Freeman and A. C. Anderson, Phys. Rev. B 34, 5684 (1986).

${ }^{15}$ R. C. Zeller and R. O. Pohl, Phys. Rev. B 4, 2029 (1971); C. C. Yu and J. J. Freeman, ibid. 36, 7620 (1987).

${ }^{16}$ J. L. Feldman, J. Q. Broughton, P. B. Allen, and F. Wooten, Bull. Am. Phys.Soc. 35, 216 (1990).

${ }^{17}$ D. G. Cahill, H. E. Fischer, T. Klitsner, E. T. Swartz, and R. 
O. Pohl, J. Vac. Sci. Technol. A 7, 1259 (1989).

${ }^{18} \mathrm{P}$. Bruench, Phonons: Theory and Experiments III (SpringerVerlag, Berlin, 1987).

${ }^{19}$ W. D. Luedtke and U. Landman, Phys. Rev. B 40, 1164 (1989).
${ }^{20}$ Defect states may increase the electronic contributions to the thermal conductivity. However, this contribution is quite small by an order of $10^{-7}$ at best using the Drude model. See the paper, K. P. Chik and K. C. Koon, Philos. Mag. B 53, 399 (1986). 